\title{
Documenting Elimination of Co-Circulating Covid-19 Clusters Using Genomics in New South Wales, Australia
}

Alicia Arnott ( $\sim$ alicia.arnott@health.nsw.gov.au )

NSW Health Pathology https://orcid.org/0000-0003-2637-8936

Jenny Draper

NSW Health Pathology: New South Wales Health Pathology

Rebecca J Rockett

Centre for Infectious Diseases and Microbiology - Public Health, Westmead Hospital

\section{Connie Lam}

Centre for Infectious Diseases and Microbiology - Public Health, Westmead Hospital

\section{Rosemarie Sadsad}

University of Sydney, Sydney Informatics Hub

\section{Mailie Gall}

NSW Health Pathology: New South Wales Health Pathology

\section{Elena Martinez}

NSW Health Pathology: New South Wales Health Pathology

\section{Roy Byun}

NSW Health: New South Wales Ministry of Health

Jennie Musto

NSW Health: New South Wales Ministry of Health

\section{Ben Marais}

Marie Bashir Institute for Infectious Diseases and Biosecurity

\section{Sharon C-A Chen}

NSW Health Pathology: New South Wales Health Pathology

Jen Kok

NSW Health Pathology: New South Wales Health Pathology

\section{Dominic E Dwyer}

NSW Health Pathology: New South Wales Health Pathology

\section{Vitali Sintchenko}

NSW Health Pathology: New South Wales Health Pathology 
Keywords: SARS-CoV-2, genomic epidemiology, bioinformatics, whole genome sequencing, Australia Posted Date: June 25th, 2021

DOI: https://doi.org/10.21203/rs.3.rs-645999/v1

License: (c) (1) This work is licensed under a Creative Commons Attribution 4.0 International License. Read Full License

Version of Record: A version of this preprint was published at BMC Research Notes on November 17th, 2021. See the published version at https://doi.org/10.1186/s13104-021-05827-x. 


\section{Abstract}

Objective: To adapt 'fishplots' to describe SARS-CoV-2 genomic cluster evolution.

Results: This novel analysis adapted the fishplot to depict the size and duration of circulating genomic clusters over time in New South Wales, Australia. It illuminated the effectiveness of interventions on the emergence, spread and eventual elimination of clusters and distilled genomic data into clear information to inform public health action.

\section{Introduction}

Since the arrival of the first COVID-19 case in Australia, the Pathogen Genomics Team at New South Wales (NSW) Health Pathology's Institute of Clinical Pathology and Medical Research (ICPMR) and the University of Sydney have implemented prospective and responsive whole genome sequencing (WGS) of confirmed SARS-CoV-2 infections. A strong positive correlation between genomics-informed clusters and epidemiologically linked cases was rapidly established, and prospective SARS-CoV-2 WGS transitioned from a novel tool with unproven real-time relevance to an essential element of NSW's COVID-19 public health response [1]. Key to this transformation and the uptake of genomics by public health professionals has been the development of novel methods to enable better visualisation and clear communication of genomic results.

In this correspondence we describe the adaptation and application of 'fishplots' to display COVID-19 genomic cluster architecture and evolution over time. The fishplot is a variant of a streamgraph, originally developed to enable visualisation of clonal tumour evolution [2]. We have adapted fishplot analysis to describe SARS-CoV-2 virus population dynamics over time, generating an "epi-fishplot" analogous to a stacked epidemiological curve (Fig. 1) [3]. This analysis simultaneously depicts the relative size and duration of circulating SARS-CoV-2 genomic clusters, defined by comparison of SARS-CoV-2 consensus sequences, over the course of the epidemic. While a conventional phylogenetic tree represents genomic relatedness between individual cases, epi-fishplot analysis enables the timing and impact of key public health interventions to be easily identified and monitored, such as the successful elimination of circulating genomic clusters within the NSW population as a result of effective public health policies (Fig. 1).

\section{Main Text}

The state of NSW is Australia's most populous with 8 million residents accounting for $31 \%$ of the Australian population, $65 \%$ of whom live in Greater Sydney. Since the Australian border was closed to all non-Australian travellers on March 15th and Australians returning from overseas have been required to complete a mandatory 14-day quarantine in designated hotels since March 28th, NSW has accommodated over half of the travellers returning to Australia by air. Despite this and at the time of writing (March 28th, 2021), NSW accounted for just 17\% $(n=5094)$ of Australia's confirmed COVID-19 
cases with no extensive local transmission of any strains linked to foreign travellers returning after March 15th. In total, overseas acquired cases restricted to hotel quarantine outweighed locally acquired cases, accounting for $59 \%$ and $41 \%$, respectively. The multidisciplinary public health response to COVID-19 implemented in NSW is co-ordinated by the Public Health Emergency Operations Centre within the NSW Ministry of Health (NSW Health) [4]. Key to terminating local SARS-CoV-2 transmission chains has been the active case finding and contract tracing conducted by NSW Health for each laboratory-confirmed case, which includes generating a SARS-CoV-2 genomic sequence to monitor its spread.

The integration of genomics into routine public health response has addressed key limitations of conventional epidemiological methods including poor or incorrect case recall, and confirmation of contentious or tenuous links. Furthermore, genomics has been instrumental in the timely identification of links between cases for which epidemiological links were not immediately apparent, supplementing conventional contact tracing methods and informing targeted public health resource allocation. In NSW, the integration of genomics into routine public health practice includes prioritisation of clinical samples for rapid sequencing, weekly verbal and written reports to NSW Health and customised on-demand reports for urgent, high-priority cases. To date, the Pathogen Genomics Team has generated and shared 1144 complete SARS-CoV-2 genomes representing $28 \%$ of all confirmed COVID-19 cases in NSW. On the basis of epidemiological information provided by NSW Health and specific single nucleotide polymorphism (SNP) profiles [1], these genomes have been classified into 53 genomic clusters. The median duration, i.e. circulation in the community, of identified genomic clusters was two weeks, although this was highly variable (range: 1-16 weeks) with clusters consisting of a median of three cases (range: 2-204 cases; Fig. 1). Epidemiological data also enabled the Pathogen Genomics Team to report which cases belong to specific transmission chains, the detail of which is described in regular reports that overlay conventional phylogenetic trees with infographics representing the supplied epidemiological data. An epi-fishplot is generated as part of the report to provide a population-level overview of SARS-CoV-2 clusters co-circulating in the community, illuminating the effectiveness of public health measures on the emergence, spread and eventual elimination of transmission chains within the local population (Fig. 1) [4].

Our data illustrates the two epidemiologically distinct waves of SARS CoV-2 infections experienced in NSW to date, the peaks of which occurred in late March and July, respectively. The first wave resulted from multiple independent introductions of genomically distinct viruses by overseas travellers prior to the closure of international borders on March 15th. Sustained local transmission of introduced strains was the exception and the median duration in weeks for the 17 first wave clusters identified ( $\geq 5$ cases) was 4 (range: 1-10: Fig. 1). By mid-May, local transmission of all clusters identified in the first wave had been eliminated, despite continued importation of overseas acquired cases into the NSW hotel quarantine system. This state of elimination, without significant or sustained local transmission (no clusters consisting of $\geq 5$ cases) was maintained for the following two months (Fig. 1).

Genome sequencing confirmed that the second wave was seeded by a domestic importation from neighbouring state, Victoria, in early July. The interstate resident travelled to NSW immediately prior to the 
border between the two states closing for the first time in 100 years in order to prevent spill-over from Victoria into NSW. Confirming that importation was the source of the initial second wave cluster (NSW33.0: Fig. 1), and not undetected community transmission, provided important reassurance that the public health measures in place were effective. The initial infection event occurred at a large licensed venue situated in close proximity to a highway traversing the east coast of Australia, which facilitated infection of multiple individuals and enabled this strain to initiate multiple transmission chains amongst the local population (Fig. 1). Genome sequencing confirmed that public health measures had eliminated the NSW33.0 cluster by mid-September, and the NSW33.1 subcluster by early November.

Globally, the COVID-19 pandemic has necessitated extraordinary and often innovative public health responses to prevent widespread virus transmission. The high frequency of asymptomatic infections or subclinical disease and comparatively limited genomic diversity of circulating strains has exposed limitations of conventional epidemiological and genomic approaches when deployed in isolation to contain the spread of SARS-CoV-2. Our experience of rapidly integrating epidemiological and genomic data into actionable information demonstrates that local elimination is achievable if stringent public health measures are maintained.

Genomic sequencing is a powerful public health tool that provides a unique level of oversight and addresses critical limitations of conventional epidemiological methods [5]. The epi-fish approach described above can be equally applied to other pathogens of public health importance subjected to integrated genomic surveillance. The successful integration of genomics into routine public health response relies on a strong working partnership between public health practitioners and laboratory professionals, with evidence synthesis and visualisation in order to distil complex genomic data into the information which can guide and benchmark public health actions.

\section{Limitations}

A sequence could not be obtained from every confirmed case of COVID-19 detected in NSW during the study period. Sequences were reliably obtained from samples with a diagnostic PCR Ct value $\leq 30$ only. However, the proportion of sequenced cases (28\%) was high compared to that reported by most countries around the world and more than sufficient to achieve the primary goal of this study which was to demonstrate the application and utility of the Epifish package.

\section{Abbreviations}

NSW: New South Wales

WGS: whole genome sequencing

ICPMR: Institute of Clinical Pathology and Medical Research

SNP: single nucleotide polymorphism 
PCR: polymerase chain reaction

Ct: cycle threshold

\section{Declarations}

\section{Ethics approval and consent to participate}

Clinical specimens were routinely processed at the ICPMR and deemed not research. A non-research determination for this project was granted by Health Protection NSW since it was a designated communicable disease control activity.

\section{Availability of data and material}

The consensus genome sequences included in this study are all available from GISAID (www.gisaid.org). A full list of genomes and their corresponding IDs can be found in Additional File 1. The Epifish package for $\mathrm{R}$ is available from Github (https://github.com/learithe/epifish).

\section{Funding}

This study was supported by the Prevention Research Support Program funded by the NSW Ministry of Health and the National Health and Medical Research Council Centre for Research Excellence in Emerging Infectious Diseases [no. GNT1102962]. V.S. is supported by an Australian National Medical Research Council fellowship [APP1123879]. The funders of this study had no role in study design, data collection, data analysis and interpretation, or writing of the article

\section{Acknowledgements}

We acknowledge the Sydney Informatics Hub and use of the University of Sydney's high-performance computing cluster, Artemis. We thank the NSW Health Pathology partner laboratories, ACT Pathology, Douglass Hanly Moir, Australian Clinical Laboratories and Laverty Pathology for referring samples for genomic surveillance. Expert advice and epidemiological information provided by the NSW public health surveillance units at NSW Health are also gratefully acknowledged. The authors are indebted to all researchers and their organizations who have shared SARS-CoV-2 genome data on GISAID.

\section{Consent for publication}

Not applicable 


\section{Competing interests}

The authors declare that they have no competing interests

\section{Author contributions}

Data generation/collection/analysis was performed by AA, JD, RR, CL, RB and JM. Bioinformatic analyses and support was performed by RR, RS, MG and EM. JD performed R coding. Drafting the manuscript was performed by AA, JD, BM, JK, DD, SC and VS. The final manuscript was approved by all authors.

\section{References}

1. Rockett RJ, Arnott A, Lam C, Sadsad R, Timms V, Gray KA, et al. Revealing COVID-19 transmission in Australia by SARS-CoV-2 genome sequencing and agent-based modelling. Nat Med. 2020;26(9):1398-404. doi:10.1038/s41591-020-1000-7.

2. Miller CA, McMichael J, Dang HX, Maher CA, Ding L, Ley TJ, et al. Visualizing tumor evolution with the fishplot package for R. BMC Genom. 2016;17(1):880. doi:10.1186/s12864-016-3195-z.

3. Draper J. Epifish package for R, https://github.com/learithe/epifish.

4. McAnulty JM, Ward K. Suppressing the Epidemic in New South Wales. N Engl J Med. 2020;382:e74. doi:10.1056/NEJMc2011592.

5. Deng X, Gu W, Federman S, du Pleiss L, Pybus OG, Faria NR, et al. Genomic surveillance reveals multiple introductions of SARS-coV-2 into Northern California. Science. 2020;369(6503):682-7. doi:10.1126/science.abb9263.

\section{Figures}



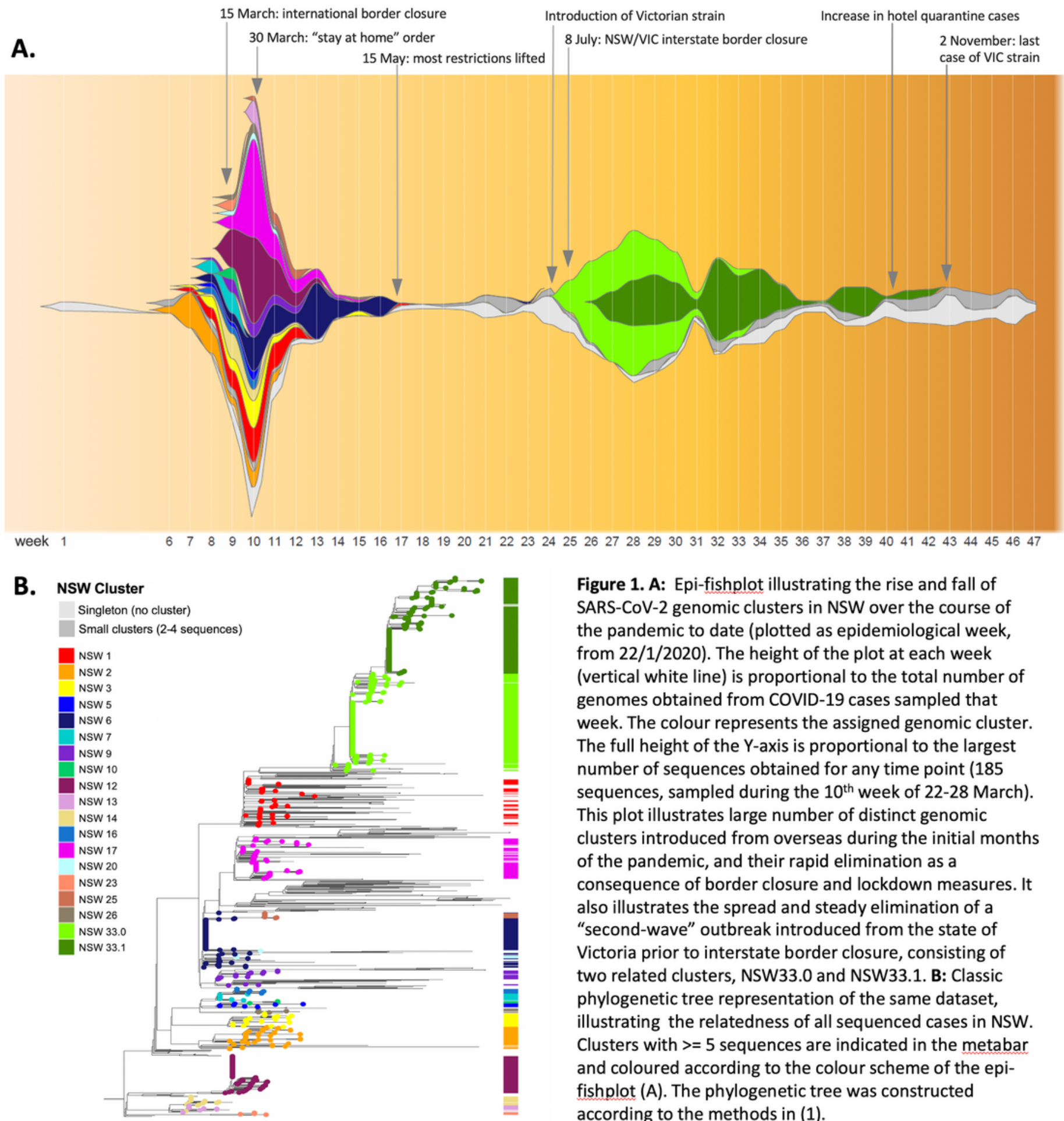

Figure 1. A: Epi-fishplot illustrating the rise and fall of SARS-CoV-2 genomic clusters in NSW over the course of the pandemic to date (plotted as epidemiological week, from 22/1/2020). The height of the plot at each week (vertical white line) is proportional to the total number of genomes obtained from COVID-19 cases sampled that week. The colour represents the assigned genomic cluster. The full height of the $\mathrm{Y}$-axis is proportional to the largest number of sequences obtained for any time point (185 sequences, sampled during the $10^{\text {th }}$ week of $22-28 \mathrm{March}$ ). This plot illustrates large number of distinct genomic clusters introduced from overseas during the initial months of the pandemic, and their rapid elimination as a consequence of border closure and lockdown measures. It also illustrates the spread and steady elimination of a "second-wave" outbreak introduced from the state of Victoria prior to interstate border closure, consisting of two related clusters, NSW33.0 and NSW33.1. B: Classic phylogenetic tree representation of the same dataset, illustrating the relatedness of all sequenced cases in NSW. Clusters with >= 5 sequences are indicated in the metabar and coloured according to the colour scheme of the epifishplot (A). The phylogenetic tree was constructed according to the methods in (1).

\section{Figure 1}

Epi-fishplot illustrating the rise and fall of SARS-CoV-2 genomic clusters in NSW over the course of the pandemic to date (plotted as epidemiological week, from 22/1/2020). (a) The height of the plot at each week (vertical white line) is proportional to the total number of genomes obtained from COVID-19 cases sampled that week. The colour represents the assigned genomic cluster. The full height of the Y-axis is proportional to the largest number of sequences obtained for any time point (185 sequences, sampled 
during the 10th week of 22-28 March). This plot illustrates large number of distinct genomic clusters introduced from overseas during the initial months of the pandemic, and their rapid elimination as a consequence of border closure and lockdown measures. It also illustrates the spread and steady elimination of a "second-wave" outbreak introduced from the state of Victoria prior to interstate border closure, consisting of two related clusters, NSW33.0 and NSW33.1. (b) Classic phylogenetic tree representation of the same dataset, illustrating the relatedness of all sequenced cases in NSW. Clusters with $\geq 5$ sequences are indicated in the metabar and coloured according to the colour scheme of the epifishplot (a). The phylogenetic tree was constructed according to the methods1.

\section{Supplementary Files}

This is a list of supplementary files associated with this preprint. Click to download.

- Additionalfile1.xlsx 